\title{
Yoghurt and curd cheese addition to wheat bread dough: Impact on in vitro starch digestibility and estimated glycemic index
}

\author{
Carla Graça, Anabela Raymundo, Isabel de Sousa* \\ Universidade de Lisboa, Instituto Superior de Agronomia, LEAF (Linking Landscape Environment Agriculture and Food) Research Center, Tapada da \\ Ajuda, 1349-017 Lisboa, Portugal
}

A R T I C L E I N F O

\section{Keywords:}

Yoghurt

Curd cheese

Wheat bread

Digestibility

Glycemic index

\begin{abstract}
A B S T R A C T
The effect of yoghurt and curd cheese additions on pasting properties, starch digestibility and estimated glycemic index of wheat bread were studied. Yoghurt and curd cheese incorporations (6\% up to 25\% w/w) promoted considerable changes on starch performance based on gelatinization and final dough consistency properties. These changes led to a significant impact on starch digestibility, reducing significantly the rapidly digestiblestarch while increasing the resistant starch. The estimated glycemic index reflected the changes promoted on starch performance from both dairy products addition, at higher level tested (25\%): a significant reduction of around $30 \%$ for yoghurt bread and $38 \%$ for curd cheese bread, was obtained, resulting in medium to low (55-69) glycemic index breads. Correlations were found between pasting properties, starch digestibility and glycemic index, revealing that the effects observed are proportional to the levels of dairy products added. Microstructure images of the starch granules supported these findings.
\end{abstract}

\section{Introduction}

Bread has long been part of the human diet and nutrition for thousands of years (Smith, Daifas, El-Khoury, Koukoutsis, \& El-Khoury, 2004), and is also rich in high-level of rapidly digestible starch which can impact on the glycemic response (Shumoy, Van Bockstaele, Devecioglu, \& Raes, 2018). In this sense and considering that health issues have been a top priority for the consumers, it is important to search for new production strategies and/or new bakerýs ingredients to reduce the glycemic response of starchy-rich foods.

Accordingly, dairies can be considered potential bakerýs ingredients, since they are reported as low glycemic index products (GI < 55) (Wolter, Hager, Zannini \& Arendt, 2014), in addition to rich protein sources with essential amino acids profile, that can be alternative strategies to reduce the glycemic response of the bakery goods. Yoghurt (Yg), is considered the most popular dairy product (DP) worldwide for its nutritional and health benefits, since it is a source of protein (casein), exopolysaccharides (EPS), vitamins (B2, B6, and B12), and minerals (such as $\mathrm{Ca}, \mathrm{P}$, and $\mathrm{K}$ ), representing an alternative for

healthier bakery products (Sharafi et al., 2017; Graça et al., 2019; 2020).

Curd cheese (Cc) is a cheese co-product, obtained by the thermal denaturation and subsequent precipitation of the soluble whey protein (WP), essentially composed by $\beta$-lactoglobulin, $\alpha$-lactalbumin and bovine serum albumin (20-25\% of the milk proteins). A considerable impact on protein and mineral profile enhancement on wheat bread, by $\mathrm{Cc}$ additions, was earlier reported (Graça et al., 2019).

Recent evidence has shown that the enrichment of the wheat bread formulation by protein-rich ingredients, can reinforce the interaction between starch and proteins, further enhanced by baking process, limiting the accessibility of $\alpha$-amylase to starch granules and probably reducing the glycemic response (Fardet et al., 2006; Chung, Lin, Hoover, Warkentin, \& Vandenberg, 2008). Furthermore, the presence of fiber and/or other microbial exopolysaccharides, in addition to starchprotein interactions, may also contribute to reducing the GI (Fardet et al., 2006; Lynch, Coffey, \& Arendt, 2018).

Therefore, the inclusion of yoghurt and curd cheese as bakery ingredients can be an approach to control the enzymatic attack on starch via encapsulation mechanisms by protein-starch interaction, impacting on starch gelatinization performance during the baking process.

This work aimed to study the influence of plain yoghurt or fresh curd cheese additions ( $6 \%$ up to $25 \%$ ), to reduce the glycemic response of the wheat bread. The impact of $\mathrm{Yg}$ or Cc on starch performance, by heatingcooling cycles, was firstly assessed. The physical integrity of starch granules structure, after heating-cooling cycles, was evaluated by scanning electron microscopy. Subsequently, the starch digestibility of the obtained wheat bread was evaluated by an in vitro digestion model, and the glycemic index was calculated. Correlations between

\footnotetext{
${ }^{*}$ Corresponding author.

E-mail address: isabelsousa@isa.ulisboa.pt (I.d. Sousa).
} 
pasting properties, starch digestibility and glycemic index were tested to acquire additional information about the processes involved.

\section{Materials and methods}

\subsection{Raw materials}

Bread was prepared using commercial wheat flour Type 65 from Granel Cereal Milling Industry, Alverca, Portugal (13.5\% moisture, $11.5 \%$ protein and $25 \%$ of carbohydrates, $\mathrm{w} / \mathrm{w}$ ).

The plain yoghurt $(\mathrm{Yg})$ used is a product from Nestlé LongaVida, Portugal (88.5\% moisture, $4 \%$ protein, $5.5 \%$ carbohydrates, w/w). The dry extract of $\mathrm{Yg}$ was determined from the Standard Portuguese Method: NP.703-1982 (Standard Portuguese Norm), corresponding to $11.5 \%$ of dry matter.

The fresh curd cheese (Cc) used was from Lacticínios do Paiva, Lamego, Paiva, Portugal (75\% moisture, $11 \%$ protein, 3\% carbohydrates, w/w). The dry extract of Cc was determined from the Standard Portuguese Method: NP.3544-1987 (Standard Portuguese Norm), corresponding to $25 \%$ of dry matter.

Commercial white crystalline saccharose (Sidul, Santa Iria de Azóia, Portugal), sea salt (Vatel, Alverca, Portugal), baker's dry yeast (Fermipan, Lallemand Iberia, SA, Setubal, Portugal), and SSL-E481sodium stearoyl-2 lactylate (Puratos, Portugal) were also used.

\subsection{Bread dough preparation}

The bread dough was prepared according to Graça et al. (2019) $2.4 \%$ yeast and $0.6 \%$ sugar were added to warm (distilled) water and dissolved well; $1.0 \%$ salt, $0.3 \%$ SSL and $6 \%, 18 \%$ or $25 \%$ of dairy products were incorporated into $58.5-53.0 \%$ wheat flour and mixed with $31.3-19.0 \%$ (distilled) water, according to each bread formulation, to complete $36.6 \%$ of wheat flour water absorption, previously optimized (Table 1. Supplementary Materials).

The bread dough preparation was performed in triplicate by randomized sampling to cover the variability of the raw materials used.

\subsection{Pasting properties}

The effect of Yg and Cc addition on starch rheology behaviour of the wheat dough was evaluated using the microdoughLab equipment applying the mixing and heating-cooling cycles, according to following setting conditions were: sample homogenization for $30 \mathrm{~s}$, mixing curve at $30{ }^{\circ} \mathrm{C}$ for $360 \mathrm{~s}$, heating from 30 to $95{ }^{\circ} \mathrm{C}$ for $390 \mathrm{~s}$, holding at $95{ }^{\circ} \mathrm{C}$ for $60 \mathrm{~s}$, cooling down to $30^{\circ} \mathrm{C}$ for $390 \mathrm{~s}$, at similar temperature rate $\left(0.17^{\circ} \mathrm{C} / \mathrm{s}\right)$. Paddle speed was $63 \mathrm{rpm}$ for the first $30 \mathrm{~s}$, and then set steady at $120 \mathrm{rpm}$ for running the analysis.

The dough consistency values (expressed in torque units, $\mathrm{mNm}$ ) produced by kneading the wheat dough is measured, in real time, to study its physic behavior, delivering the follow parameters (AACC, 5460.01): water absorption (\%) - the percentage of water required to reach the optimal dough consistency, dough development time (C1) or maximum dough consistency, which is driven by the gluten matrix; protein weakening $\left(\mathrm{C}_{2}\right)$, at this stage the temperature starts to rise, and the lower consistency is attained, due to the heat denaturation of proteins; starch gelatinization $\left(\mathrm{C}_{3}\right)$, under constant shear and increase of temperature the starch granules starts to swell, and will break, loose amylose and gelatinize, increasing the torque values; cooking stability (C4) or the minimum torque reached at this phase of cooking, in which the amylase activity is dominating as well as the stabilization of the amylose network; starch gelling $\left(\mathrm{C}_{5}\right)$ or final consistency peak torque produced by further cooling, in which gelation taking the lead with possible retrogradation/crystallization of amylose and continuous increase of torque and final consistency (Huang et al., 2010).

Triplicates were performed to ensure reproducible results.

\subsection{In vitro starch hydrolysis}

\subsubsection{Total starch (TS)}

The total starch in bread crumb samples: control, Yg and Cc breads, was determined enzymatically following the method described by Goni et al. (1997).

All the incubation steps were performed in a controlled shaking water - bath equipment (Thermo-Scientific- Model: 2871, Waltham, MA, U.S.A).

Ground bread (50 mg) was dispersed in $6 \mathrm{ml}$ of $2 \mathrm{M} \mathrm{KOH}$ and shaken (30 min at room temperature); $3 \mathrm{ml}$ of $0.4 \mathrm{M}$ sodium acetate buffer ( $\mathrm{pH}$ 4.75) and $60 \mu \mathrm{l}$ of amyloglucosidase (330oU/mL) (EC-3.2.1.3, SigmaAldrich, Chemical Company, St Louis, MO, USA) were added; the suspension was incubated ( $45 \mathrm{~min}$ at $60^{\circ} \mathrm{C}$ ), under controlled shaking water-bath. Triplicates were performed.

2.4.1.1. Resistant starch (RS). Resistant starch was estimated according to the methodology described by Goni et al. (1997).

Grounded bread sample (100 g) was incubated (6o min at $40{ }^{\circ} \mathrm{C}$ ) with a pepsin solution, from porcine gastric mucosa (EC-3.4.23.1, Sigma-Aldrich, Chemical Company, St Louis, MO, USA) $(1 \mathrm{~g} / 10 \mathrm{ml}$ buffer KCL-HCL: $40 \mathrm{OOOU} / \mathrm{mL}$ ), to protein interference removal. Starch was hydrolyzed by adding pancreatic $\alpha$-amylase (EC-3.2.1.1, SigmaAldrich, Chemical Company, St Louis, MO, USA) (40 mg $\alpha$-amylase per $\mathrm{ml}$ Tris-maleate buffer: $200 \mathrm{U} / \mathrm{mL}$ ) and incubated ( $16 \mathrm{~h}$ at $\left.37^{\circ} \mathrm{C}\right)$; obtained samples were washed three times with deionized water, and the pellet was separated by centrifugation to further digestion with $\mathrm{KOH}$ $4 \mathrm{M}$; this solution (at $\mathrm{pH} 4.75$ ) was incubated (45 $\mathrm{min}$ at $60{ }^{\circ} \mathrm{C}$ ) with amyloglucosidase $(3300 \mathrm{U} / \mathrm{ml})$.

Total and resistant starches were measured as glucose release, using a glucose oxidase-peroxidase (GODPOD) reagent kit (K-Glox, Megazyme Bray, Co. Wicklow, Ireland). The absorbance (510 nm) was

\section{Table 1}

Variation of the rheology parameters of wheat starch by the addition of dairy products, evaluated during mixing and heating-cooling circles on microdoughLab analysis.

\begin{tabular}{|c|c|c|c|c|c|c|c|}
\hline & & $\mathrm{C} 1$ & $\mathrm{C} 2$ & $\mathrm{C}_{3}$ & & $\mathrm{C}_{4}$ & $\mathrm{C}_{5}$ \\
\hline Samples & WA (\%) & $\mathrm{DD}(\mathrm{mNm})$ & $\mathrm{PW}(\mathrm{mNm})$ & $\mathrm{SG}(\mathrm{mNm})$ & GT $\left({ }^{\circ} \mathrm{C}\right)$ & $\mathrm{CS}(\mathrm{mNm})$ & $\mathrm{FV}(\mathrm{mNm})$ \\
\hline $\mathrm{CD}$ & $52.4 \pm 0.5^{\mathrm{a}}$ & $128.0 \pm 2.9^{\mathrm{a}}$ & $77.7 \pm 1.2^{\mathrm{a}}$ & $250.0 \pm 6.0^{\mathrm{a}}$ & $83.9 \pm 0.8^{\mathrm{a}}$ & $221.7 \pm 11.0^{a}$ & $594.0 \pm 4.4^{\mathrm{a}}$ \\
\hline $\mathrm{Yg}_{6 \%}$ & $51.6 \pm 2.1^{\mathrm{a}}$ & $135.3 \pm 0.1^{\mathrm{a}}$ & $76.3 \pm 2.1^{\mathrm{a}}$ & $244.0 \pm 1.7^{\mathrm{a}}$ & $81.8 \pm 1.5^{\mathrm{a}}$ & $237.7 \pm 2.5^{\mathrm{a}}$ & $593.3 \pm 2.1^{\mathrm{a}}$ \\
\hline $\mathrm{Yg}_{18 \%}$ & $45.4 \pm 0.4^{b}$ & $131.3 \pm 4.2^{\mathrm{a}}$ & $70.1 \pm 3.2^{a}$ & $220.5 \pm 2.2^{\mathrm{a}}$ & $81.8 \pm 1.5^{\mathrm{a}}$ & $197.6 \pm 6.1^{\mathrm{a}}$ & $589.0 \pm 3.1^{\mathrm{a}}$ \\
\hline $\mathrm{Yg}_{25 \%}$ & $39.3 \pm 1.2^{\mathrm{d}}$ & $132.7 \pm 2.3^{\mathrm{a}}$ & $27.3 \pm 3.2^{b}$ & $185.3 \pm 4.6^{b}$ & $79.7 \pm 1.8^{\mathrm{a}}$ & $65.5 \pm 2.1^{b}$ & $600.0 \pm 7.6^{a}$ \\
\hline $\mathrm{Cc}_{6 \%}$ & $51.8 \pm 2.3^{\mathrm{a}}$ & $130.7 \pm 0.2^{\mathrm{a}}$ & $73.1 \pm 1.2^{\mathrm{a}}$ & $217.5 \pm 5.1^{\mathrm{a}}$ & $78.2 \pm 1.7^{\mathrm{a}}$ & $106.3 \pm 9.6^{\mathrm{d}}$ & $551.5 \pm 3.6^{\mathrm{a}}$ \\
\hline $\mathrm{Cc}_{18 \%}$ & $49.6 \pm 0.1^{\mathrm{ab}}$ & $127.0 \pm 2.0^{a}$ & $71.7 \pm 0.6^{a}$ & $179.7 \pm 7.1^{\mathrm{b}}$ & $73.5 \pm 0.7^{b}$ & $97.1 \pm 5.1^{\mathrm{d}}$ & $495.0 \pm 6.9^{b}$ \\
\hline $\mathrm{Cc}_{25 \%}$ & $50.7 \pm 0.6^{\mathrm{ab}}$ & $127.3 \pm 5.8^{\mathrm{a}}$ & $69.7 \pm 3.5^{\mathrm{a}}$ & $112.7 \pm 1.2^{c}$ & $69.4 \pm 1.5^{c}$ & $54.0 \pm 1.5^{\mathrm{e}}$ & $86.7 \pm 6.7^{c}$ \\
\hline
\end{tabular}

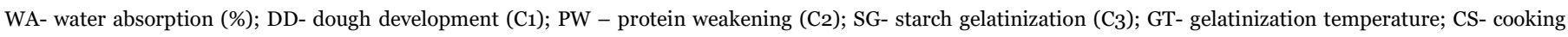
stability (C4); FV- final viscosity $\left(\mathrm{C}_{5}\right)$. Different letters within the same column are statistically different $(\mathrm{p}<0.05)$. 

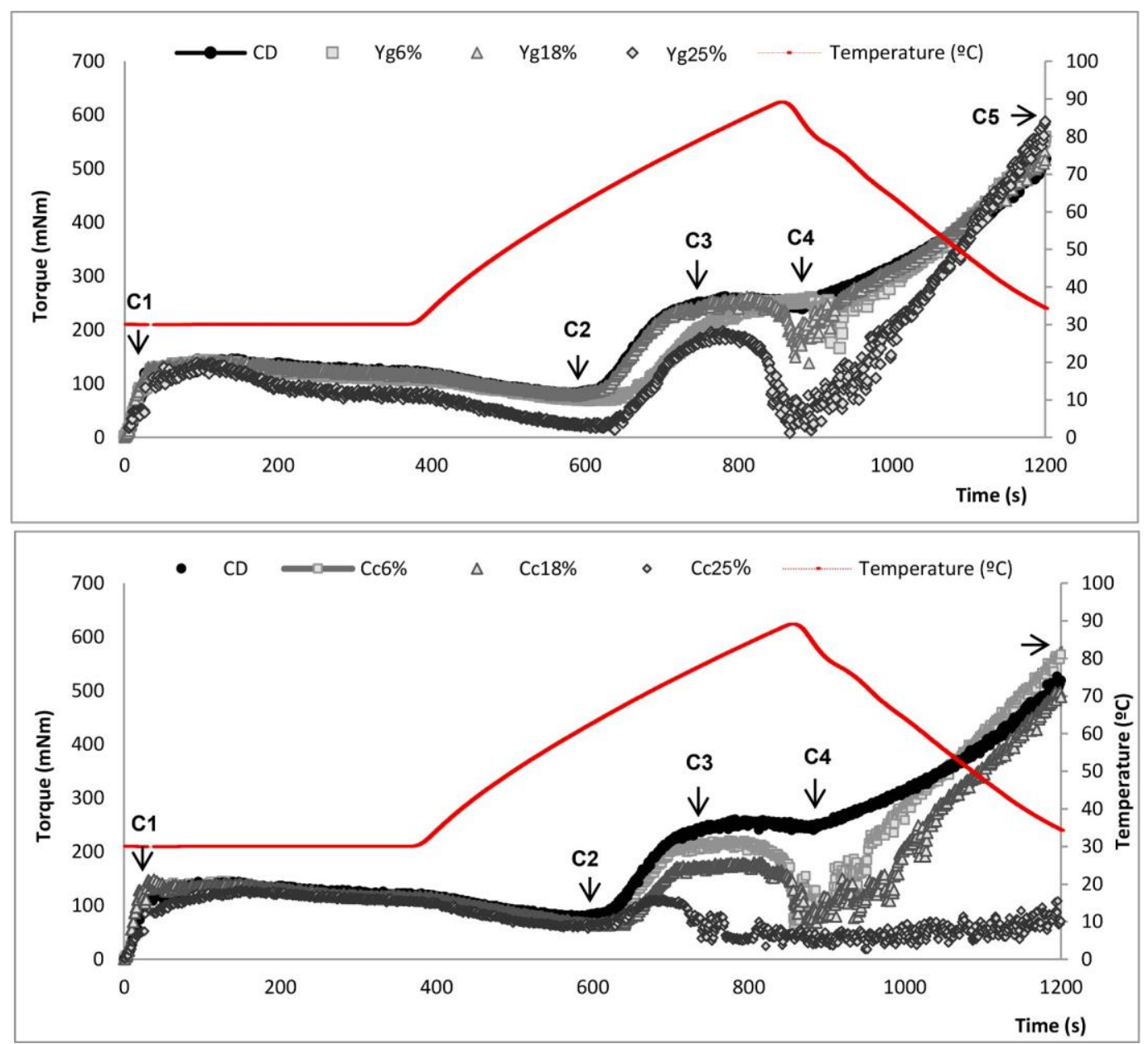

Fig. 1. Effect of $\mathrm{Yg}(\mathrm{A})$ and $\mathrm{Cc}(\mathrm{B})$ addition on pasting properties of wheat starch, determined by MicroDoughLab analysis: CD- control wheat dough; C1- dough development time; $\mathrm{C}_{2}$ - protein weakening; $\mathrm{C}_{3}$ - starch gelatinization; $\mathrm{C}_{4}$ - cooking stability; $\mathrm{C}_{5}$ - final viscosity.

measured using a microplate reader (Spectramax, Bio-TEK, MultiDetection Synergy HT, UK). Starch was calculated as glucose (mg) $\times 0.9$ (conversion factor). Triplicates were performed.

2.4.1.2. In vitro starch digestibility and estimated glycemic index. To evaluate the effect of the DP addition on the starch digestibility and

to predicted glycemic index of the bread, an in vitro starch hydrolysis based in the procedure described by Goni et al. (1997), was applied. Three phases were simulated: 1) to chewing phase, bread sample (100 $\mathrm{mg}$ ) was milled; 2) to gastric phase, the grounded bread was dispersed in $10 \mathrm{ml}$ of $0.1 \mathrm{M}$ KCL-HCL buffer (pH 1.5) and $200 \mu \mathrm{l}$ of

pepsin solution ( $1 \mathrm{~g} / 10 \mathrm{ml} \mathrm{KCL-HCL}$ buffer), followed by incubation (60 min at $\left.40{ }^{\circ} \mathrm{C}\right) ; 3$ ) to pancreatic phase, $25 \mathrm{ml}$ of $0.1 \mathrm{M}$ of tris-maleate

buffer ( $\mathrm{pH}$ 6.9) and $5 \mathrm{ml}$ of a pancreatic $\alpha$-amylase solution ( $3 \mathrm{U} / \mathrm{ml}$ trismaleate buffer) was added; followed by incubation $\left(37^{\circ} \mathrm{C}\right)$. Triplicates of $1 \mathrm{ml}$ were taken at every thirty minutes $(30-180 \mathrm{~min})$ and placed into boiling water $(5 \mathrm{~min})$ to inactivate the enzyme reaction; followed by refrigeration conditions $\left(4{ }^{\circ} \mathrm{C}\right)$ until the end of incubation time $(180$ $\mathrm{min})$. For each aliquot taken, $3 \mathrm{ml}$ of $0.4 \mathrm{M}$ sodium acetate buffer $(\mathrm{pH}$ 4.75) and $60 \mu \mathrm{l}$ of amyloglucosidase (3300 $\mathrm{U} / \mathrm{ml}$ ) were added, followed by incubation ( $45 \mathrm{~min}$ at $60^{\circ} \mathrm{C}$ ); the volume was adjusted to $10 \mathrm{ml}$ with distilled water, mixed and centrifuged $(3000 \times \mathrm{g} / 10 \mathrm{~min})$; the supernatant was taken for glucose determination.

The glucose content was measured using a glucose oxidase-peroxidase (GODPOD) kit as described for total and resistant starch procedure. Triplicates were performed.
A nonlinear model, as expressed by Eq. (1), was employed to describe the kinetics of starch hydrolysis, where $\mathrm{C}$ was the concentration at $t$ time, Coowas the equilibrium concentration, $k$ was the kinetic constant and $t$ was the time.

$C=C_{\infty}\left(1-e^{-k t}\right)$

The hydrolysis index (HI), was obtained from Eq. (2), dividing the area under hydrolysis curve of the breads (AUC $0-180 \mathrm{~min}$ ) by the area under curve of the reference food (fresh white wheat bread) over the same period time.

$H I=\frac{\text { AUC of product }}{\text { AUC Reference food }} \times 100$

The in vitro digestion kinetics was calculated in accordance with the procedure established by Goni et al (1997). 
C. GThate estimation of glycemic indices (eGI) were calculated according to Eq. (3), proposed earlier by Goni et al. (1997).

$\mathrm{eGI}=(0.549 \times \mathrm{HI})+39.71$

\subsection{Microstructure of the dough - Starch granules size}

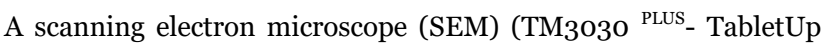
Microspcope- Hitachi, Japan) was used to observe the starch granules physical integrity of control dough, Yg and Cc doughs, after the heating-cooling cycles applied. Samples were placed on the specimen holder, dried automatically by the equipment, and the freezing model was applied $\left(-14{ }^{\circ} \mathrm{C}\right)$. The observations were analysed at $800 \times$ of magnification, with scale bar of $100 \mu \mathrm{m}$. Triplicates were performed. 


\subsection{Statistical analysis}

The experimental results were statistically analysed by determining the average value, standard deviation, and the significance level was set at $95 \%$, for each parameter evaluated. Statistical analysis (RStudio, Version 1.1.423) was performed by applying variance analysis, the one factor (ANOVA), and post-hoc comparisons (Tukey test). The experimental data was fitted to nonlinear model and the relation between continuous variables was carried out by correlation matrix using the Pearson product moment correlation distribution to significant $p$ $<0.05$.

\section{Results and discussion}

\subsection{Pasting properties of wheat starch}

The impact of $\mathrm{Yg}$ and Cc incorporation to wheat dough on gluten and starch performance, was studied based on pasting properties microdoughLab procedure.

From Fig. 1 the heating-cooling cycles obtained show the different impacts of the Yg (A) and Cc (B) on gluten proteins and on starch behavior, compared with control dough.

The impact observed by Yg addition is much lower than that observed for the Cc addition, most probably due to the different structure of the proteins involved on the starch-gluten matrix as well as the differences on protein content (Bertolini et al., 2005; Noisuwan et al., 2008). Some peak values reflect the influence of the $\mathrm{Yg}$ and $\mathrm{Cc}$ on pasting properties of doughs (Fig. 1), and these parameters are summarized in Table 1. The incorporation of $\mathrm{Yg}$ on wheat dough, up to $18 \%$ $(\mathrm{w} / \mathrm{w})$ of addition, has no significant effect $(\mathrm{p}>0.05)$ on protein weakening (C2), starch gelatinization ( $\left.\mathrm{C}_{3}\right)$, cooking stability $\left(\mathrm{C}_{4}\right)$ and final consistency $\left(\mathrm{C}_{5}\right)$, with values similar to the control ones. Forhigher levels of $\mathrm{Yg}$ tested $(25 \%, \mathrm{w} / \mathrm{w})$ a significant decrease $(\mathrm{p}<0.05)$ on $\mathrm{C} 2$, meaning further protein weakening $(27.3 \pm 3.2 \mathrm{mNm})$, com- pared to control dough $(77.7 \pm 1.2 \mathrm{mNm})$, was observed, most probably due to the dilution effect of the gluten proteins (Graça et al., 2018; 2019). The lubrication effect of gluten by the exopolysaccharides (EPS) produced by lactic acid bacteria in Yg can be also considered (Lynch et al., 2018) to explain this observed torque reduction. A sig- nificant decrease ( $\mathrm{p}<$ 0.05) on peak consistency ( $\left.\mathrm{C}_{3}\right)$, by starch ge- latinization $(185.3 \pm 4.6$ $\mathrm{mNm}$ ), was also registered, compared to control dough $(250.0 \pm 6.0$ $\mathrm{mNm}$ ), representing a reduction of $26 \%$. During cooking stability $\left(\mathrm{C}_{4}\right)$, a phase separation process was observed, promoting a significant decrease in torque units of $\mathrm{Yg}$ dough $(65.5 \pm 2.1 \mathrm{mNm})$ compared to control dough $(221.7 \pm 11.0 \mathrm{mNm})$, that represents $70.5 \%$ of torque variation. However, during cooling stage, the $\mathrm{Yg}$ dough structure reinforces with values of final torque ( $\left.\mathrm{C}_{5}\right)$ slightly higher $(600.0 \pm 7.6$ $\mathrm{mNm}$ ) than the control dough $(594.0 \pm 4.4 \mathrm{mNm})$. This result suggests that the caseins and the EPS may have strong interactions with starch molecules, which upon cooling increase the consistency by becoming entrapped in the gelled matrix, by EPS-amylose-casein-gluten interactions. Probably, this be- haviour was mediated by the depletion flocculation mechanism (phase separation phenomena), as earlier described in similar complex systems (Nunes, Raymundo, \& Sousa, 2006; Considine, Noisuwan, Hemar, Wilkinson, Bronlund, \& Kasapis, 2011).

According to the results obtained for Cc addition, at all levels tested, on stages of dough development $(\mathrm{C} 1)$ and protein weakening $(\mathrm{C} 2)$, no significant effects were observed, presenting values like those obtained to control. However, as the temperature increases, the addition of different CC levels promoted significant changes on starch performance, in terms of starch gelatinization, cooking stability and final consistency. Considering higher levels of Cc tested $(25 \%, \mathrm{w} / \mathrm{w})$, where the impact was more evident, a strong effect on starch performance was noticed: starting from starch gelatinization, a significant decrease on peak consistency (112.7 $\pm 1.2 \mathrm{mNm}$ ) was registered, compared to control dough $(250.0 \pm 6.0 \mathrm{mNm})$, representing a reduction of $55 \%$. The cooking stability $\left(\mathrm{C}_{4}\right)$ and the final consistency $\left(\mathrm{C}_{5}\right)$ were also negatively affected, representing a decrease in torque units of $49.2 \%$ and $84.3 \%$, respectively. These results suggested that denatured Cc proteins have a thermodynamic incompatibility with hydrolyzed starch molecules, hindering a matrix structuration during cooling, which could be attributed not only to the dilution effect of the starch but also to different causes like: i) the starch started to swell but due to the high competition for available water, from denatured Cc proteins, swelling was hindered (Noisuwan et al., 2008) by insufficient hydration; ii) starch-protein interactions, reducing the volume portion occupied by swelled granules in dough and the concentration of the amylose molecules (Chung et al., 2008).

In Cc doughs, the starch should be less damaged than in the $\mathrm{Yg}$ dough, as it can be seen by the lower values of torque for starch gelatinized $\left(\mathrm{C}_{3}\right)$ and final peak consistency $\left(\mathrm{C}_{5}\right)$, therefore less available.

According to the results obtained, it can be stated that the different nature and structure of the dairy proteins added in the form of the $\mathrm{Yg}$ and Cc, promoted different effects on starch rheology behaviour. These changes were probably a result of complex dough structure modification, which can be attributed to the macromolecules interactions, and/ or phase separation mechanisms, due to the incompatibility phenomena between the molecular chains involved on dough matrix.

\subsection{In vitro starch digestibility of wheat bread}

The impact of yoghurt and curd cheese addition, at different levels, on in vitro starch digestibility was evaluated based on the procedure well described earlier by Goni et al. (1997).

The levels (g/10o g) of total starch (TS), resistant starch (RS) and digestible starch (DS) of the developed breads were determined and are summarize in Table 2.

The TS content in the dairy breads ranged from 60.0 to $50.0 \mathrm{~g} / 100 \mathrm{~g}$ for Yg breads and from 50.0 to $40.0 \mathrm{~g} / 100 \mathrm{~g}$ for Cc breads, compared to control bread (65.0 g/100 g). Resistant starch (RS) content varied among the dairy breads with a range of 5.0-7.0 g/100 $\mathrm{g}$ for Yg breads and 6.1-7.6 g/100 g for Cc breads, compared to the control one ( $4.4 \mathrm{~g} /$ $100 \mathrm{~g}$ ). Comparing the control bread and maximum levels of both dairy products tested $(25 \%, \mathrm{w} / \mathrm{w})$ the increase on RS content was higher in Cc breads $(73.0 \%)$ than in $\mathrm{Yg}$ breads $(60 \%)$.

This significant increase in RS content, could be attributed to the effect on the starch performance, probably by entrapping the granules and hindering the complete gelatinization, possibly due to the establishment of starch-proteins interaction further enhanced during the baking stage (Fardet et al., 2006; Chung, Lin, Hoover, Warkentin, \& Vandenberg, 2008). In addition, the competition to available water should also be considered to justify this starch behaviour, since a strong depletion on starch gelatinization was observed, which can be associated to the insufficient starch water uptake. RS acts as a soluble fiber, since it is not absorbed in small intestine but is fermented in the large intestine by bacteria microbiome, which is considered as beneficial to human health (Lehmann \& Robin, 2007). These results agree with those

Table 2

Total Starch (TS), Resistant Starch (RS) and Digestible Starch (DS) of control (CB), yoghurt (Yg) and curd cheese (Cc) breads.

\begin{tabular}{llll} 
Samples & TS $(g / 100 \mathrm{~g})$ & RS $(\mathrm{g} / 100 \mathrm{~g})$ & DS $(\mathrm{TS}-\mathrm{RS})(\mathrm{g} / 100 \mathrm{~g})$ \\
\hline $\mathrm{CB}$ & $65.0 \pm 1.1^{\mathrm{a}}$ & $4.4 \pm 0.4^{\mathrm{a}}$ & $60.3 \pm 0.3^{\mathrm{a}}$ \\
$\mathrm{Yg}_{6 \%}$ & $60.0 \pm 4.1^{\mathrm{a}}$ & $5.0 \pm 0.5^{\mathrm{a}}$ & $55.0 \pm 2.2^{\mathrm{a}}$ \\
$\mathrm{Yg}_{18 \%}$ & $59.0 \pm 1.3^{\mathrm{a}}$ & $5.2 \pm 1.0^{\mathrm{a}}$ & $54.0 \pm 1.5^{\mathrm{a}}$ \\
$\mathrm{Yg}_{25} \%$ & $49.4 \pm 0.9^{\mathrm{b}}$ & $7.0 \pm 1.1^{\mathrm{b}}$ & $43.0 \pm 2.0^{\mathrm{b}}$ \\
$\mathrm{Cc}_{6 \%}$ & $50.0 \pm 2.7^{\mathrm{b}}$ & $6.1 \pm 0.3^{\mathrm{b}}$ & $47.0 \pm 1.8^{\mathrm{b}}$ \\
$\mathrm{Cc}_{18 \%}$ & $47.3 \pm 1.3^{\mathrm{b}}$ & $6.4 \pm 0.5^{\mathrm{b}}$ & $41.0 \pm 2.6^{\mathrm{b}}$ \\
$\mathrm{Cc}_{25 \%}$ & $40.0 \pm 3.5^{\mathrm{c}}$ & $7.6 \pm 0.6^{\mathrm{c}}$ & $31.3 \pm 2.1^{\mathrm{c}}$ \\
\hline
\end{tabular}

Different letters within the same column are significantly different $(\mathrm{p}<0.05)$. 
obtained in pasting properties where a strong negative effect on starch gelatinization was observed for higher levels of Cc addition tested.

In terms of digestible starch (DS), both $\mathrm{Yg}$ and $\mathrm{Cc}$ addition at the higher levels tested $(25 \%, \mathrm{w} / \mathrm{w})$ promoted a significant reduction(p $<0.05$ ) of $29.0 \%$ and $48.0 \%$, respectively. From the results pre- sented, the dilution effect of the starch (TS and DS) is evident, and theseresults are also in agreement with those obtained on pasting properties. The starch fractions variation observed can be attributed to the inter- action between dairy proteins (caseins from Yg and denatured whey proteins from $\mathrm{Cc}$, additions) and starch molecules, restricting the granules swelling and extension of the starch gelatinization. This resultsin a lower susceptibility to enzymatic attack, which may be partially responsible for the low starch digestibility observed (Chandrashekar \& Kirleis, 1988; Reshmi et al., 2017).

As it is well-known, the rate of starch digestion is the main factor that influences the glucose release and absorption during digestion (Jenkins et al., 1982). Based on in vitro hydrolysis the digestible starch can be distinguished into two different fractions: rapidly digested starch (RDS) - hydrolyzed within the first 30 min of digestion and slowly digested starch (SDS) - digested within the following $100 \mathrm{~min}$. RDS has a significant impact on glycemic response, whereas the SDS presents low impact, due to the slow glucose release over the hydrolysis time (Englyst et al., 1996).

The influence of both DP on digestible starch fractions (RDS and SDS) was evaluated, and the results are illustrated at Fig. 2. The Yg (A) and $\mathrm{Cc}$ (B) additions had a positive influence on digestible starch fractions: from DP additions of $18 \%(\mathrm{w} / \mathrm{w})$, a significant reduction(p $<0.05$ ) on RDS was observed, and a remarkable effect was achieved for higher level of both DP tested (25\%, w/w), representing a reduction of $62 \%$ for $\mathrm{Yg}$ and $73 \%$ for Cc breads, compared to the control bread. Since the starch gelatinization, during baking process, is the main mechanism responsible for the RDS fraction increase (Shumoy et al., 2018), these results should reflect the dilution effect on starch granules and/or the reinforcement of starch- protein interaction pro- moted by the $\mathrm{Yg}$ and $\mathrm{Cc}$ addition, driving to a inhibition of the

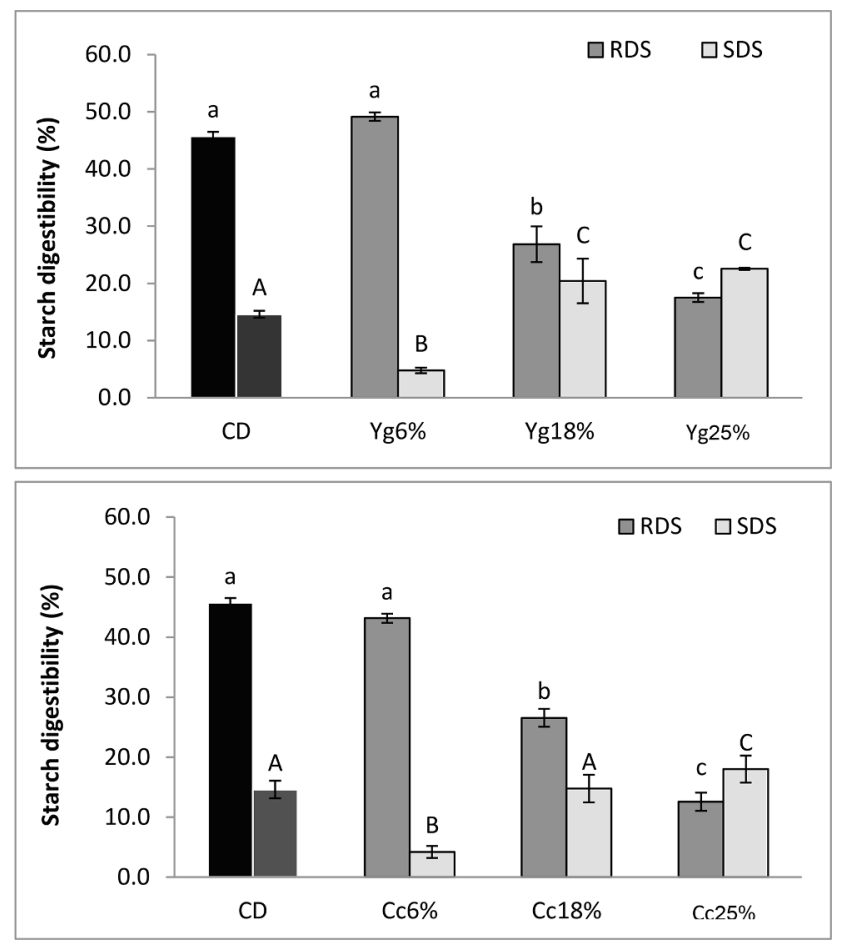

Fig. 2. Effect of the $\mathrm{Yg}(\mathrm{A})$ and $\mathrm{Cc}(\mathrm{B})$ addition on digestible starch fractions: rapidly digestible starch (RDS) and slowly digestible starch (SDS). Different letters in each bar indicate significant differences $(\mathrm{p}<0.05)$. gelatinization process, that may led to a significant reduction on RDS.

Based on SDS fraction and considering the higher level of the $\mathrm{Yg}$ and Cc additions tested (25\%, w/w), an increase of $54.2 \%$ on Yg bread was attained, whereas for Cc bread only $23.3 \%$ of increase was observed, compared to the control bread. This lower increase of SDS to Cc bread could be attributed to the negative effect of $\mathrm{Cc}$ on starch gelatinization, driving to starch granules becoming more resistance to enzymatic attack, increasing the resistant starch content (Table 2).

In general, higher values of SDS are desirable over the RDS, due to nutritional aspects related to the slowly digestible of the starch in the small intestine, inducing a gradual insulin production after ingestion (Jenkins et al., 1982). Similar results were obtained by other authors (Giuberti, Gallo, Cerioli, Fortunati, \& Masoero, 2015) using bean flour to produce gluten-free pasta.

Based on the results presented, it was noticeable that the impact of DP on starch performance, mainly on gelatinization and final consistency values, is reflected on digestible starch fractions. Therefore, a possible correlation between the addition of DP on pasting properties and in vitro starch hydrolysis was investigated by Pearsońs correlation analysis $(\mathrm{p}<0.05)$. A negative correlation was found between pasting properties with both DP additions: starch gelatinization $\left(\mathrm{r}_{\mathrm{Yg}}=-0.971\right.$; $\left.\mathrm{r}_{\mathrm{Cc}}=-0.979\right)$, cooking stability $\left(\mathrm{r}_{\mathrm{Yg}}=-0.928\right)$ and final consistency $\left(\mathrm{r}_{\mathrm{Yg}}=-0.688 ; \mathrm{r}_{\mathrm{Cc}}=-0.989\right)$. A positive correlation was observed for Cc addition on cooking stability $\left(\mathrm{r}_{\mathrm{Cc}}=0.574\right)$. In terms of starch digestibility, a negative correlation was also obtained between RDS and both DP ( $\left.\mathrm{r}_{\mathrm{Yg}}=-0.846 ; \mathrm{r}_{\mathrm{Cc}}=-0.992\right)$, whereas a positive correlation was observed for SDS $\left(\mathrm{r}_{\mathrm{Yg}}=0.671 ; \mathrm{r}_{\mathrm{Cc}}=0.887\right)$ and $\mathrm{RS}\left(\mathrm{r}_{\mathrm{Yg}}=0.855\right.$; $\mathrm{r}_{\mathrm{Cc}}=0.915$ ), supporting the relations aforementioned.

These correlations telling that the effect observed on pasting properties is proportional to the quantity of DP added.

Considering the possible relation between pasting properties and digestible starch fraction, for both DP tested, RDS is positively correlated with starch gelatinization $\left(\mathrm{r}_{\mathrm{Yg}}=0.947 ; \mathrm{r}_{\mathrm{Cc}}=0.988\right)$ and final consistency $\left(\mathrm{r}_{\mathrm{Yg}}=0.985 ; \mathrm{r}_{\mathrm{Cc}}=0.896\right)$, whereas SDS showed a negative correlation (starch gelatinization: $\mathrm{r}_{\mathrm{Yg}}=-0.826 ; \mathrm{r}_{\mathrm{Cc}}=-0.825$; final consistency: $\left.\mathrm{r}_{\mathrm{Yg}}=-0.891 ; \mathrm{r}_{\mathrm{Cc}}=-0.911\right)$.

The impact of $\mathrm{Yg}$ and $\mathrm{Cc}$ addition on starch gelatinization and on final consistency should be considered to explain the results obtained, since the starch swelling and subsequent structure breakdown increase the accessibility of enzyme attack into the granules, consequently, increasing the RDS content. The gelatinized starch is more susceptible to hydrolysis generating higher RDS content and lower SDS and RS contents (Englyst et al., 1996; Chung et al., 2008).

The results presented show that the Yg and Cc additions, at higher level, promoted significant changes on starch physical behaviour which were reflected on in vitro starch digestibility, contributing to reduce significantly the RDS fraction, increasing the SDS and RS fractions, and this must have an impact in reducing the glycemic response of bread.

\subsection{Hydrolysis kinetics and estimated glycemic index of the breads}

The influence of the Yg and Cc additions on starch hydrolysis kinetics was studied and the glycemic index of the obtained wheat breads was calculated.

Nonlinear parameters of the starch hydrolysis kinetics were obtained by fitting the experimental data (Eq. (1)) that describes well the impact of dairy products on starch digestion dynamic (Goni et al.,1997). These fitted parameters included equilibrium concentration $(\mathrm{C} \infty)$, kinetics constant $(k)$, hydrolysis index (HI) and estimated gly- cemic index (eGI), that are summarize at Table 3.

From Table 3, it can be observed that the incorporation of $\mathrm{Yg}$ and $\mathrm{Cc}$ had a significant $(\mathrm{p}<0.05)$ impact on equilibrium concentration reduction $(\mathrm{C} \infty)$, varying from 52.7 to $32.2 \%$ for Yg breads and from 47.6 to 20.0\% for Cc additions, compared to control bread (54.3\%). These results are in line with those obtained for the starch fractions discussed above, where a considerable reduction on total starch was registered. 
Table 3

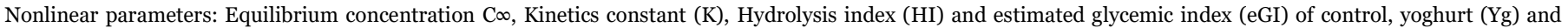
curd cheese (Cc) breads.

\begin{tabular}{|c|c|c|c|c|c|}
\hline Samples & $\mathrm{C} \infty$ & $k$ & $\mathrm{R}^{2}$ & $\mathrm{HI}$ & eGI* \\
\hline $\mathrm{CB}$ & $54.3 \pm 1.4^{\mathrm{a}}$ & $0.065 \pm 0.004^{a}$ & 0.960 & $100.0 \pm 0.0^{a}$ & $100.0 \pm 0.0$ \\
\hline $\mathrm{Yg}_{6 \%}$ & $52.7 \pm 0.6^{b}$ & $0.104 \pm 0.018^{b}$ & 0.996 & $100.3 \pm 0.9^{\mathrm{a}}$ & $94.7 \pm 0.5^{\mathrm{a}}$ \\
\hline $\mathrm{Yg}_{18 \%}$ & $47.5 \pm 0.9^{c}$ & $0.034 \pm 0.002^{c}$ & 0.986 & $81.0 \pm 0.5^{b}$ & $84.1 \pm 0.3^{b}$ \\
\hline $\mathrm{Yg}_{25 \%}$ & $32.2 \pm 0.9^{\mathrm{d}}$ & $0.019 \pm 0.002^{c}$ & 0.957 & $47.5 \pm 0.4^{c}$ & $65.7 \pm 0.2^{c}$ \\
\hline $\mathrm{Cc}_{6} \%$ & $47.6 \pm 1.4^{c}$ & $0.077 \pm 0.001^{a}$ & 0.998 & $88.1 \pm 2.1^{\mathrm{d}}$ & $88.0 \pm 1.1^{\mathrm{d}}$ \\
\hline $\mathrm{Cc}_{18 \%}$ & $39.5 \pm 1.0^{\mathrm{e}}$ & $0.035 \pm 0.003^{c}$ & 0.986 & $67.4 \pm 0.5^{\mathrm{e}}$ & $76.6 \pm 0.3^{\mathrm{e}}$ \\
\hline $\mathrm{Cc}_{25 \%}$ & $20.0 \pm 0.3^{f}$ & $0.033 \pm 0.006^{c}$ & 0.975 & $33.8 \pm 0.8^{\mathrm{f}}$ & $58.2 \pm 0.4^{\mathrm{f}}$ \\
\hline
\end{tabular}

*eGI $=(0.549 \times \mathrm{HI})+39.71$; Different letters within the same column are statistically different $(\mathrm{p}<0.05)$.

The kinetics constant $(k)$, indicative of the enzymatic hydrolysis rate in the early stage, in general showed decreasing values with increasing levels of $\mathrm{Yg}$ and $\mathrm{Cc}$ : $\mathrm{Yg}$ breads ranging from 0.104 to 0.019, and for Cc breads varying between 0.077 and 0.033 compared to control bread (0.065). Lower values of $k$ suggest that the increase of dairy product additions to dough are probably promoting higher resistance to enzymatic starch hydrolysis, and the decrease in digestibility, may be explained by the starch-protein interaction, are limiting the starch digestion, during enzymatic hydrolysis (Chung et al., 2008).

These results are in line with those obtained on digestible starch fractions, where a significant reduction on RDS and an increase on RS were registered. Similar results were obtained by Barine \& Yorte (2016), by incorporation of "Amala" and Plantain on bread formula- tions.

Hydrolysis index (HI) calculated from the rate of starch hydrolysis over time and the respective estimated glycemic index (eGI) are also presented in Table 3. For Yg and Cc breads, up to $18 \%(\mathrm{w} / \mathrm{w})$ of addition, higher values of $\mathrm{HI}$ resulted in higher eGI, ranging from 94.7 to 84.1 to Yg breads and 88.0-76.6 to Cc breads, compared to control bread (100). The lowest HI and eGI was recorded at higher levels of $\mathrm{Yg}$ and $\mathrm{Cc}(25 \%, \mathrm{w} / \mathrm{w})$ tested, with eGI of 65.7 and 58.2, representing a reduction of $31.0 \%(\mathrm{Yg})$ and $38.4 \%(\mathrm{Cc})$, respectively. GI of foods can vary between $\leq 55$, and 56-69 and $\geq 70$, that are classified as low, medium, and high GI, respectively (Atkinson et al., 2008; Jenkins et al., 2008). According to this classification, the dairy breads developed with maximum incorporations $(25 \%, \mathrm{w} / \mathrm{w})$ can be classified in medium GI $\left(\mathrm{Yg}_{25 \%}\right)$ and almost low GI $\left(\mathrm{Cc}_{25 \%}\right)$. These results of GI could be attributed to the low RDS and high SDS and RS fractions values obtained, by in vitro starch digestibility.

The findings agree with those registered on pasting properties by the addition of DP which are reflected on digestible starch fractions and support the results obtained on glycemic index of the dairy breads. Similar relations were reported by other authors (Reshmi et al., 2017) showing that the fortification of bread with pomelo fruit segments reduced the glycemic index of the breads. Low GI foods is desirable not only for consumers with diabetes, but also for all consumers in general, since it prevents the incidence of chronic metabolic diseases (BrandMiller et al., 2009).

\section{Microstructure of the wheat dough- starch granules size and structure integrity}

Environment scanning electron microscopy (ESEM) was used to investigate the impact of the higher levels of $\mathrm{Yg}$ and $\mathrm{Cc}$ addition $(25 \%$, $\mathrm{w} / \mathrm{w}$ ) on starch granules size and structure integrity to explain and support the results, discussed above. The control, Yg and Cc doughs were collected after applying the heating/cooling cycles on microdoughLab analysis. From Fig. 3 A-C, the impact of the $\mathrm{Yg}(\mathrm{B})$ and $\mathrm{Cc}$ (C) addition on granules structure, compared to control dough (A), can be observed. Considering the control dough (A), the starch granules structures seems to be less intact on dough surface, as well as covered by a gummy structure acting such as a filling effect, aggregating the starch granular structure. This gummy structure resulted, most probably, from the amylose molecules released by the swelled starch breakdown and gelatinization during heating stage.

In the case of Yg dough (B), the ESEM image seems to show some swollen and unbreaked starch granules and, although they also look aggregated by this gummy structure, it seems to be less noticeable than in control dough. However, comparing the images of control and $\mathrm{Yg}$ doughs, slight differences can be observed.

With Cc addition (C), a different picture can be seen, there is a higher number of intact starch granules preserved, and less aggregated, compared to control and $\mathrm{Yg}$ doughs. In addition, the gummy structure around swollen starch granules are much less perceptible, as in control and Yg dough, suggesting that the gelatinization process was negatively affected by $\mathrm{Cc}$ addition.

These results agree with those obtained on pasting properties evaluation, where a significant negative influence on starch gelatinization, either for $\mathrm{Yg}$ and $\mathrm{Cc}$ addition, was observed.

The ESEM images obtained could partially explain and support the results obtained by the addition of the $\mathrm{Yg}$ and $\mathrm{Cc}$ and their impact on pasting properties, on in vitro starch digestibility as well as on the GI values of the dairy breads.

\section{Conclusion}

Considering the importance of reducing the glycemic index of the bread, this study was conceived to evaluate the incorporation of yoghurt and curd cheese on wheat bread, as an alternative approach to reduce the glycemic response. The findings showed that higher additions $(25 \%, w / w)$ of yoghurt and curd cheese negatively affected the starch gelatinization performance, and this effect was reflected on in vitro starch digestibility. A significant reduction on rapidly digestible starch was observed, while resistant starch increased, for both Yg and Cc breads, being stronger on the latter. In line with these results, are the estimated values of the glycemic index from the dairy breads, resulting in Yg breads with medium GI (55-69) and Cc breads with almost low GI $(\leq 55)$.

Improvements observed in the glycemic response of the wheat dairy breads, can probably be associated not only to the dilution of starch, promoted by the dairy products additions, but also to further starchprotein interaction enhanced by baking, limiting the gelatinization process and the enzymatic attack to starch granules. The microstructure images obtained for starch granules surface, where higher integrity of the starch granules was observed for Cc dough, give some additional support to these findings.

Correlations were found between pasting properties, starch digestibility and glycemic index, meaning that the effects observed are proportional to the quantity of the dairy products added.

In summary, the incorporation of $\mathrm{Yg}$ and Cc showed to be an interesting strategy to reduce the glycemic response of the wheat bread. 


\section{A- Control dough}
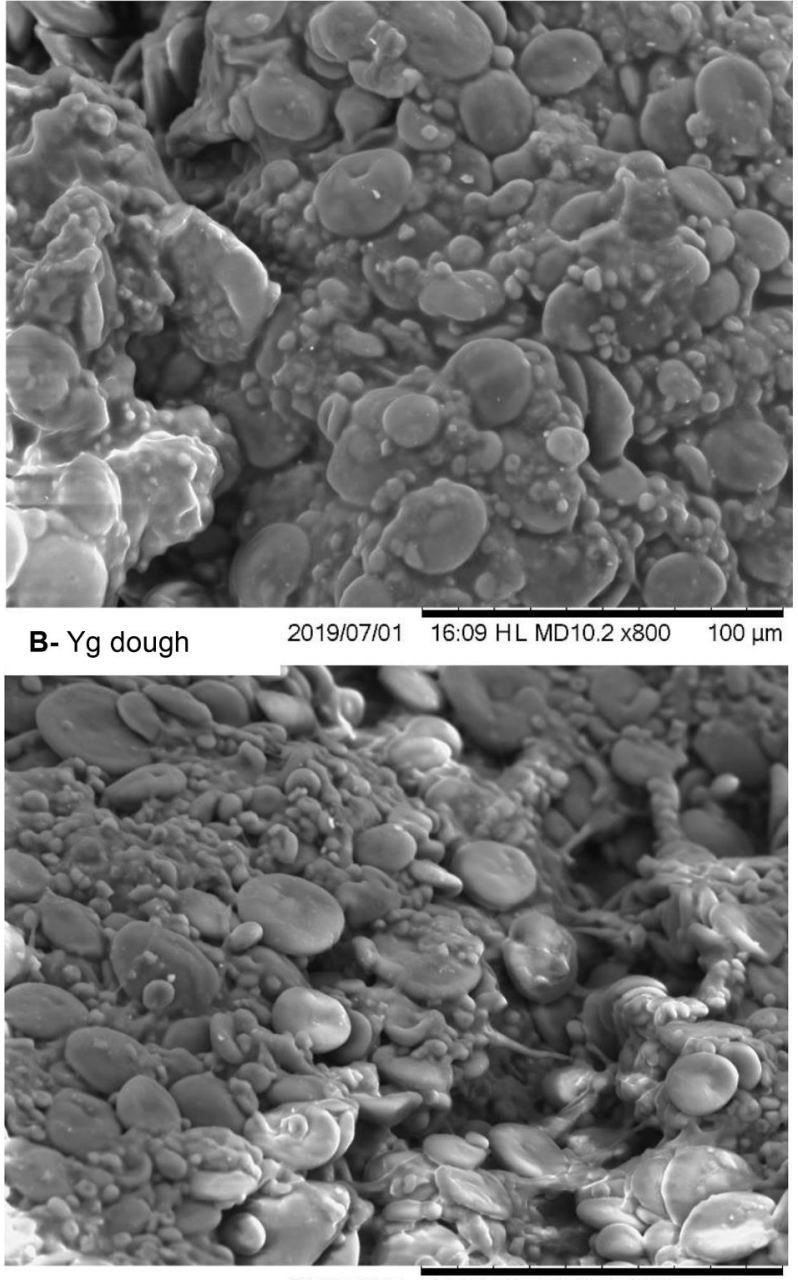

C- Cc dough

$2019 / 07 / 0511: 54$ HL MD10.2 x800 $100 \mu \mathrm{m}$

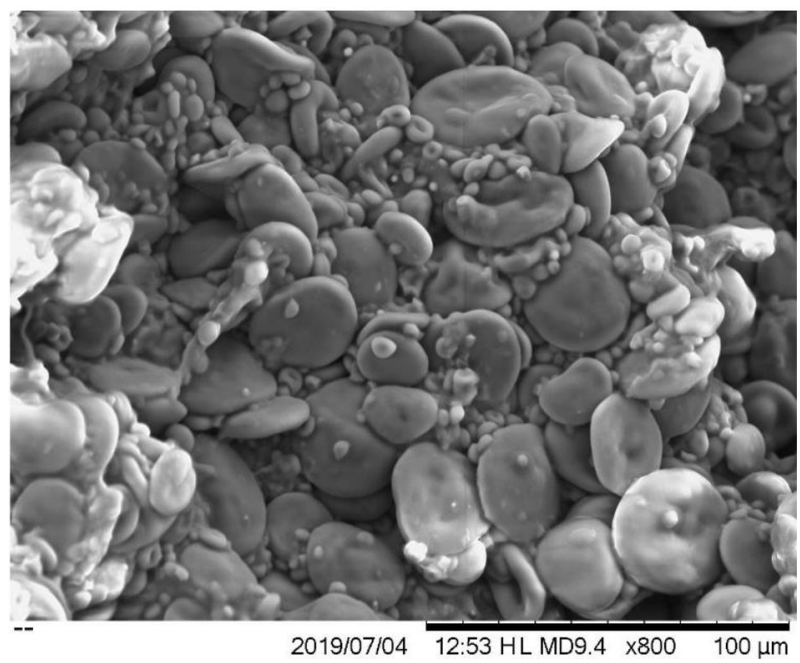

Fig. 3. Scanning electron micrographs (80ox of magnification, scale bar $=100 \mu \mathrm{m})$ : A- control dough; B- yoghurt dough and C-curd cheese dough $(25 \%$, $\mathrm{w} / \mathrm{w})$, evaluated after the heating-cooling cycles by microdoughLab analysis.

\section{Declaration of Competing Interest}

The authors declare that they have no known competing financial interests or personal relationships that could have appeared to influence the work reported in this paper.

\section{Acknowledgement}

This work was supported and financed by the University of Lisbon [Doctoral Grant reference:C10781w], and by the Portuguese Foundation for Science and Technology (FCT) through the research unit UID/AGR/04129/2020 (LEAF).

\section{Appendix A. Supplementary data}

Supplementary data to this article can be found online at https:// doi.org/10.1016/j.foodchem.2020.127887.

\section{References}

Atkinson, F. S., Foster-Powell, K., \& Brand-Miller, J. C. (2008). International tables of glycemic index and glycemic load values. Diabetes Care, 31, 2281-2283.

Barine, K. K. D., \& Yorte, G. S. (2016). In vitro starch hydrolysis and prediction of glycemic index (PGI) in "Amala" and plantain based baked products. Journal of Food Research, 5, 73-80.

Bertolini, A. C., Creamer, L. K., Eppink, M., \& Boland, M. (2005). Some rheological properties of sodium caseinate-starch gels. Journal of Agricultural and Food Chemistry, 53, 2248-2254.

Brand-Miller, J. C., Stockmann, K., Atkinson, F., Petocz, P., \& Denyerx, G. (2009). Glycemic index, postprandial glycemia, and the shape of the curve in healthy subjects: analysis of a database of more than 1000 foods. The American Journal of Clinical Nutrition, 89(1), 97105 .

Chandrashekar, A., \& Kirleis, A. W. (1988). Influence of protein on starch gelatinization in sorghum. Cereal Chemistry, 65, 457-462.

Chung, H. J., Lin, Q., Hoover, R., Warkentin, T. D., \& Vandenberg, B. (2008). In-vitro starch digestibility expected glycemic index and thermal and pasting properties of flours from pea, lentil and chickpea cultivars. Food Chemistry, 111, 316-321.

Considine, T., Noisuwan, A., Hemar, Y., Wilkinson, B., Bronlund, J., \& Kasapis, S. (2011). Rheological investigations of the interactions between starch and milk proteins in model dairy systems: A review. Food Hydrocolloids, 25, 2008-2017.

Englyst, H. N., Kingmans, S. M., \& Gummings, J. H. (1996). Classification and measurement of nutritionally important starch fraction. European Journal of Clinical Nutrition, 46, 335-505.

Fardet, A., Leenhardt, F., Lioger, D., Scalbert, A., \& Rémésy, C. (2006). Parameters controlling the glycemic response to breads. Nutrition Research Reviews, 19, 18-25.

Giuberti, G., Gallo, A., Cerioli, C., Fortunati, P., \& Masoero, F. (2015). Cooking quality and starch digestibility of gluten free pasta using new bean flour. Food Chemistry, 175, 43-49.

Goni, I., Garcia-Alonso, A., \& Saura-Calixto, F. (1997). A starch hydrolysis procedure to estimate glycemic index. Nutrition Research, 17, 427-437.

Graça, C., Fradinho, P., Sousa, I., \& Raymundo, A. (2018). Impact of Chlorella vulgaris on the rheology of wheat flour dough and bread texture. LWT - Food Science and Technology, 89, 466-474.

Graç, C., Raymundo, A., \& Sousa, I. (2019). Wheat Bread with Dairy Products-Technology, Nutritional, and Sensory Properties. Applied. Sciences, 9, 4101.

Graça, C., Raymundo, A., \& Sousa, I. (2020). Yogurt as an Alternative Ingredient to Improve the Functional and Nutritional Properties of Gluten-Free Breads. Foods, 9, 111.

Huang, W., Li, L., Wang, F., Wan, J., Tilley, M., \& Ren, C. X. (2010). Effects of transglutaminase on the rheological and Mixolab thermomechanical characteristics of oat dough. Food Chemistry, 121, 934-939.

Jenkins, D. J. A., Ghafari, H., Wolever, T. M. S., Taylor, R. H., Barker, H. M., Feilden, H., Bowling, A. C. (1982). Relationship between the rate of digestion of foods and postprandial glycemia. Diabetologia, 22, 450-455.

Jenkins, D. J., Kendall, C. W., Nguyen, T. H., Marchie, A., Faulkner, D. A., Ireland, C., \& Singer, W. (2008). Effect of plant sterols in combination with other cholesterol lowering foods. Metabolism, 57, 130-139.

Lehmann, U., \& Robin, F. (2007). Slowly digestible starch - its structure and health implications: A review. Trends in Food Science and Technology, 18, 346-355.

Lynch, K. M., Coffey, A., \& Arendt, E. K. (2018). Exopolysaccharides producing lactic acid bacteria: Their techno-functional role and potential application in gluten-free bread products. Food Research International, 110, 52-61.

Noisuwan, A., Broland, J., Wilkinson, B., \& Hemar, Y. (2008). Effect of milk protein products on the rheological and thermal (DSC) properties of normal rice starch and waxy rice starch. Food Hydrocolloids, 22(1), 174-183.

Nunes, M. C., Raymundo, A., \& Sousa, I. (2006). Gelled vegetable desserts containing pea protein, K-carrageenan and starch. European Food Research Technology, 222, 622-628.

Reshmi, S. K., Sudha, M. L., \& Shashirekha, M. N. (2017). Starch digestibility and predicted glycemic index in the bread fortified with pomelo (Citrus maxima) fruit segments. Food Chemistry, 237, 957-965. 
Sharafi, S., Yousefi, S., \& Faraji, A. (2017). Developing an innovative textural structure for semi volume breads based on interaction of spray-dried yogurt powder and jujube polysaccharide. International Journal of Biological Macromolecules, 104, 992-1002.

Shumoy, H., Van Bockstaele, F., Devecioglu, D., \& Raes, K. (2018). Effect of sourdough addition and storage time on in vitro starch digestibility and estimated glycemic index of tef bread. Food Chemistry, 264, 34-40.
Smith, J. P., Daifas, D. P., El-Khoury, W., Koukoutsis, J., \& El-Khoury, A. (2004). Shelf life and safety concerns of bakery products - A Review. Critical Review Food Science and Nutrition, 44, $19-55$

Wolter, A., Hager, A.-S., Zannini, E., \& Arendt, E. K. (2014). Influence of sourdough on in vitro starch digestibility and predicted glycemic indices of gluten-free breads. Food and Function, 5, 564 . 